\title{
Planifier les trames vertes dans les aires urbaines : une alliance à trouver entre paysagisme et écologie
}

Planning Green Spaces in Urban Areas: A Sought-For Alliance Between

Landscape Architecture and Ecology

\section{Monique Toublanc et Sophie Bonin}

\section{OpenEdition}

Journals

Édition électronique

URL : http://journals.openedition.org/developpementdurable/9347

DOI : 10.4000/developpementdurable.9347

ISSN : 1772-9971

Éditeur

Association DD\&T

Référence électronique

Monique Toublanc et Sophie Bonin, «Planifier les trames vertes dans les aires urbaines : une alliance

à trouver entre paysagisme et écologie », Développement durable et territoires [En ligne], Vol. 3, $n^{\circ}$

2 | Juillet 2012, mis en ligne le 07 juillet 2012, consulté le 19 avril 2019. URL : http://

journals.openedition.org/developpementdurable/9347; DOI : 10.4000/developpementdurable.9347

Ce document a été généré automatiquement le 19 avril 2019

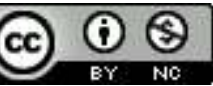

Développement Durable et Territoires est mis à disposition selon les termes de la licence Creative Commons Attribution - Pas d'Utilisation Commerciale 4.0 International. 


\title{
Planifier les trames vertes dans les aires urbaines : une alliance à trouver entre paysagisme et écologie
}

\author{
Planning Green Spaces in Urban Areas: A Sought-For Alliance Between \\ Landscape Architecture and Ecology
}

Monique Toublanc et Sophie Bonin

1 L'idée de continuité verte contenue dans l'expression trame verte est une idée nomade qui circule depuis plusieurs décennies entre différents champs, ceux de l'aménagement du territoire (notamment l'urbanisme et le paysagisme), ceux de la recherche scientifique (principalement l'écologie du paysage, mais aussi la géographie, et plus récemment la sociologie). Elle va revêtir plusieurs formulations et se transformer au fil du temps, épousant les idéologies du moment et les transformations économiques, sociales et urbanistiques de la société. Mais si les significations, les usages et le vocabulaire associés à cette idée de continuité varient selon les époques, on peut la repérer très tôt dans la pensée de la ville, dès le XIXe siècle, et d'abord chez des paysagistes, pour lesquels elle serait fondatrice de «l'art paysager» (Harter, 2002). Il est dès lors apparu intéressant de s'attacher à comprendre la contribution de la pensée du paysage à l'idée de trame verte à travers le prisme des documents d'urbanisme de deux régions : l'agglomération d'Angers ${ }^{1}$ et la plaine de Versailles ${ }^{2}$. Ce choix est motivé notamment par le souhait de comparer un contexte provincial et un contexte francilien, ce dernier étant, par rapport à la pensée de la ville, bien spécifique (capitale politique et économique, gigantisme historique à l'échelle de la France).

2 Après un rappel historique des prémices paysagers de l'idée de trame verte, nous en explorons les évolutions à travers les schémas directeurs de planification urbaine et les études des professionnels en charge de l'aménagement de ces territoires (urbanistes et paysagistes surtout), depuis les années 1960, c'est-à-dire depuis la judiciarisation de 
l'urbanisme (Loi d'orientation foncière de 1967). Nous montrons comment l'idée de préserver, voire d'aménager des continuités naturelles, a perduré et a été réinterprétée selon les significations propres à chaque époque et selon les contextes territoriaux. Filer cette idée et son cheminement dans la planification urbaine a conduit à repérer trois périodes : une période où elle est d'abord utilisée comme coupure verte, pensée comme un glacis délimitant l'urbanisation ; une période qui voit l'idée s'enrichir et se développer à travers un vocabulaire et des acceptions nouvelles, principalement sociales et paysagères, comme nous le montrerons à propos du bocage angevin; enfin, une période qui correspondrait à l'état actuel, où les fonctions attribuées à la trame verte sont devenues extrêmement multiples, mais où, peut-être de ce fait même, domine une acception écologique, qui ne parvient plus dans les documents à s'articuler aux acceptions sociales et paysagères.

\section{Les origines paysagistes d'une continuité verte aux fonctions sociales, hygiénistes et esthétiques}

3 L'idée d'un réseau vert structurant le développement des villes a des origines paysagistes, prestigieuses, remontant de façon avérée au XIX ${ }^{\mathrm{e}}$ siècle, voire au-delà. Elle correspond à un projet social porté par une double vision hygiéniste et esthétique. Aux États-Unis d'abord, le paysagiste Frederick Law Olmsted (1822-1903) développa dans la deuxième moitié du XIX ${ }^{\mathrm{e}}$ siècle l'idée des parkways, larges promenades plantées ${ }^{3}$ d'arbres permettant la circulation dans les villes (Harter, 2002 ; Fabos, 1995 et 2004 ; Décarie, 2002 ; Maumi, 2009). Il s'agissait notamment de créer et relier entre eux des parcs urbains, l'ensemble constituant le "park system » (fig. 1). L'idée lui en serait venue en 1859 lors d'une visite à Paris où il découvrit les grandes avenues d'Haussmann, et le travail d'Adolphe Alphand (Santini, 2011), l'ingénieur paysagiste qui participa à la rénovation de Paris et aménagea de nombreux parcs, squares et bois (fig. 2). Père fondateur du mouvement des greenways, Frederick Olmsted intégra alors les parcs dans la planification urbaine; il les considérait comme un élément d'organisation de la ville et de son développement ; il les désignait comme « les poumons de la cité ».

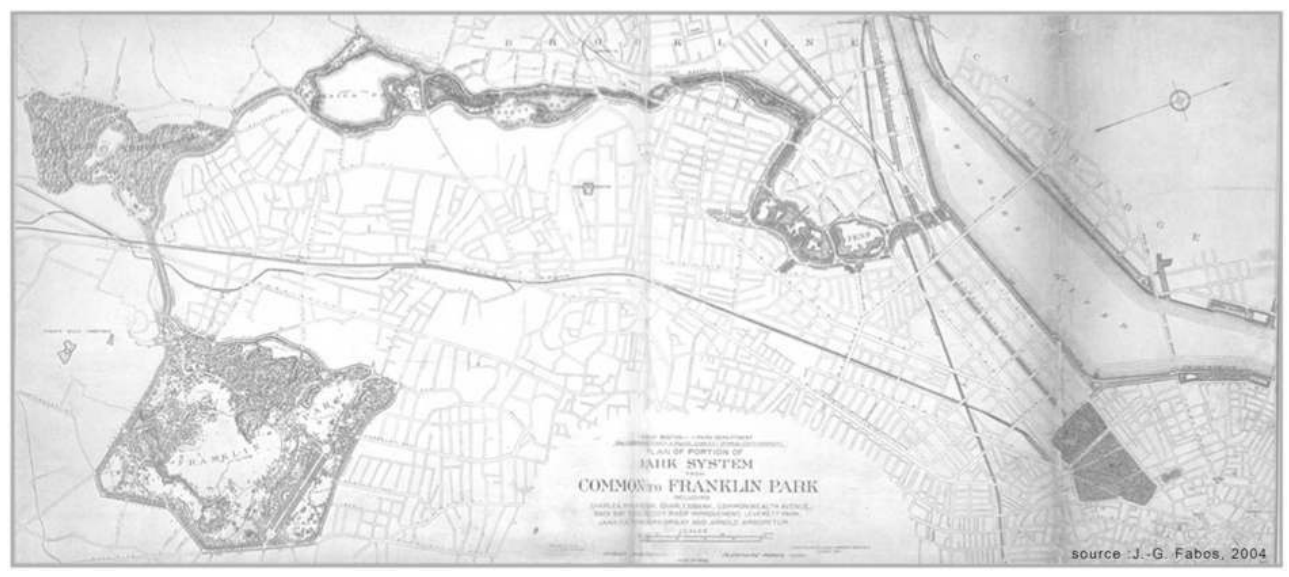


Fig. 1 : Le « park system » proposé par Frederick Law Olmsted à Boston (Massachusetts)
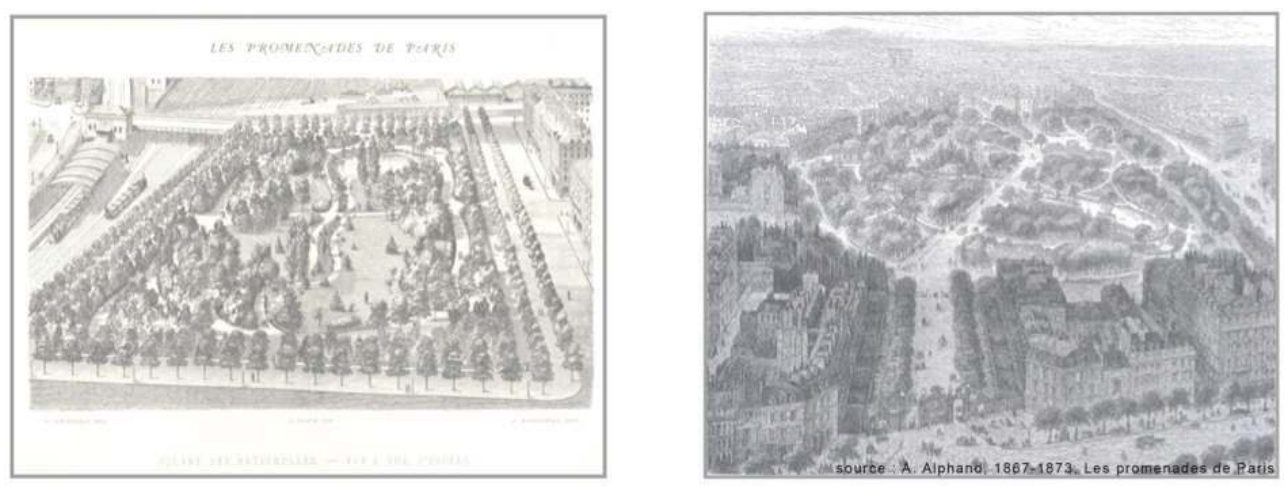

Fig. 2 : Un réseau de promenades plantées créées par Jean-Charles Adolphe Alphand

4 Ces principes, traversant une nouvelle fois l'Atlantique, furent repris par Jean Claude Nicolas Forestier (1861-1930) qui les reformula (Grandes villes et systèmes de parcs, 1908 ; réed. 2001) et chercha à les mettre en œuvre (Le Dantec, 1996 : 361-368; Pousin, 2007 : 42). Polytechnicien et ingénieur des Eaux et Forêts, devenu en 1898 conservateur en chef du secteur ouest des promenades de Paris, Jean Claude Nicolas Forestier a joué un rôle important dans l'aménagement de plusieurs grandes villes (Racine, 2002 : 148).

5 Face à la transformation des villes sous l'effet de l'industrialisation, Jean Claude Nicolas Forestier affirme en 1908 la nécessité de penser le développement urbain à partir d'un «système de parcs » qui relie une grande diversité d'espaces, à des échelles différentes, allant des "grandes réserves et paysages protégés » aux «terrains de récréation » en passant par les «avenues-promenades", les "parcs suburbains », les "grands parcs urbains », les " petits parcs», les «jardins de quartier» (Le Dantec, 1996: 365). Cette classification comprend, non seulement les parcs ou les jardins au sens classique du terme, mais également des espaces comme les bois, les pâturages, les rivières, les vallées, les rochers qui « pourront, par la continuation de leur exploitation naturelle, donner des revenus importants » (Ibidem).

6 La pensée de Forestier correspond à une représentation systémique déclinée à l'échelle de l'agglomération des grandes villes: "Le plan des villes est insuffisant, s'il n'est pas complété par un programme d'ensemble et un plan spécial des espaces libres intérieurs et extérieurs, pour le présent et l'avenir, par un système de parcs » (J.- C. Nicolas Forestier, 1908 in Le Dantec, 1996 : 365). Celui-ci correspond à un projet social porté par une double vision hygiéniste (régénération à travers le contact de la nature, santé, salubrité) et esthétique (embellissement de la ville face à l'industrialisation). « Malheureusement, il semble à bien des personnes que les parcs, les promenades publiques sont un luxe pour lequel une sage administration doit restreindre ses dépenses. Nul n'hésite à approuver les travaux les plus coûteux destinés à nous donner de l'eau pure, mais l'air et le soleil ne sont-ils pas au moins aussi nécessaires à la vie ?... Les parcs sont des éléments d'hygiène et de beauté » (Forestier, 1908). Ces espaces de nature, reliés entre eux, sont conçus pour structurer et accompagner le développement de la ville. L'enjeu est d'offrir un système de promenades aux citadins et de favoriser une meilleure circulation de l'air; il n'est pas encore question de dynamique des populations végétales ou animales. 
Pendant l'entre-deux guerres, un autre concept s'impose : celui de Green belt codifié pour la première fois en Angleterre par la loi du 29 juillet 1938 - Green belt (London and Home Counties) Act. À partir de 1955, cette idée fonde une "véritable politique des zones vertes » (Moindrot, 1961:587) dans l'aménagement des villes outre-manche. L'objectif de la ceinture verte est alors différent de celui du Parksystem: il s'agit de préserver une couronne d'espaces non bâtis autour des villes pour éviter leur agglomération. Il est désormais acquis, y compris en France, que la discontinuité urbaine est une vertu à cultiver en favorisant une continuité circulaire verte.

8 Pour autant, après la seconde guerre mondiale, l'idée d'une continuité verte irriguant la ville est encore présente dans les catégories de pensée des urbanistes-paysagistes français comme L. Soulier ou T. Leveau. Ce dernier a poursuivi la réflexion de Forestier dont il a été l'élève, puis le collaborateur à partir de 1928. En 1964, il préconise la «nécessaire pénétration des espaces verts dans la ville »; « un système d'espaces verts intérieurs qui s'intègrent, d'une manière continue, à la structure de la ville future "; " une suite de coulées vertes et de surfaces boisées [...] dans les unités de résidence »; il conseille de "proportionner et répartir la trame verte aux besoins futurs» (Leveau, 1964). Par ailleurs, toujours en 1964, dans le cadre d'une commission d'aménagement du territoire, la société des architectes diplômés par le gouvernement va défendre pour Paris l'idée d'un plan vert et d'un système d'espaces verts constituant « un filet vert extra-muros permettant de faire entrer la nature dans Paris » (Oberdoerffer, $1964: 20$ ).

En 1976, cette vision nourrit le schéma directeur d'aménagement et d'urbanisme (SDAU) de la région ${ }^{4}$ Ile-de-France (SDAURIF) mais pas celui d'Angers, ville moyenne de province (fig. 3) : le premier utilise le terme de trame verte tandis que le second l'ignore. Ce hiatus est à mettre en relation avec une tendance des années 1970 qui voient se développer l'urbanisme fonctionnel. Les clés en sont la planification et le zonage (délimitation d'aires précises pour chaque fonction: habitat /agriculture productive/ loisirs) en réponse aux problématiques du logement et du transport. Nombreux sont alors les planificateurs à envisager le «vert» comme une zone parmi d'autres et à ne pas voir dans l'idée de continuité verte matière à projet.

\section{La trame comme filet vert et paysager pour endiguer l'étalement urbain}

\section{1. À travers les schémas d'agglomération des années 1970, une conception structurante du « vert » comme coupures à l'urbanisation}

10 Sur le plan du SDAU d'Angers de 1976, les éléments naturels repérés et caractérisés prennent la forme de taches vertes isolées; il n'y a aucune idée de réseau ni même de ceinture verte. Ce schéma entérine une partition binaire du territoire:espace « aménagé » d'une part, «naturel » de l'autre. L'agriculture est classée dans la première catégorie aux côtés de l'habitat, de l'industrie et des activités tertiaires. La seconde catégorie recouvre les cours d'eau, les zones inondables, les espaces boisés et les espaces verts - type parc ou jardin urbain - aménagés à des fins récréatives (fig. 3). Pour les planificateurs, l'« espace naturel» a vocation à être protégé (vision protectionniste dominante à l'époque). Ils préconisent de conserver - ou de créer - des coupures vertes 
dans le tissu urbain afin d'empêcher le développement d'un continuum urbain. Ils distinguent les coupures "agricoles» des coupures "naturelles» (dites aussi "géographiques ») correspondant aux rivières, étangs et bois (SDAU, rapport de présentation:25-26). Ces espaces sont pensés comme un véritable outil foncier permettant d'assurer une discontinuité de l'urbanisation et de planifier un développement urbain multipolaire (un pôle principal centré sur la ville et des pôles secondaires situés en périphérie). Leur fonction écologique n'est pas ou peu mise en avant (cf. infra). Quant à leur utilité sociale, elle n'est envisagée que pour les coupures vertes dites «naturelles » qui, pour cette raison, sont perçues comme des «aires de loisirs » potentielles.

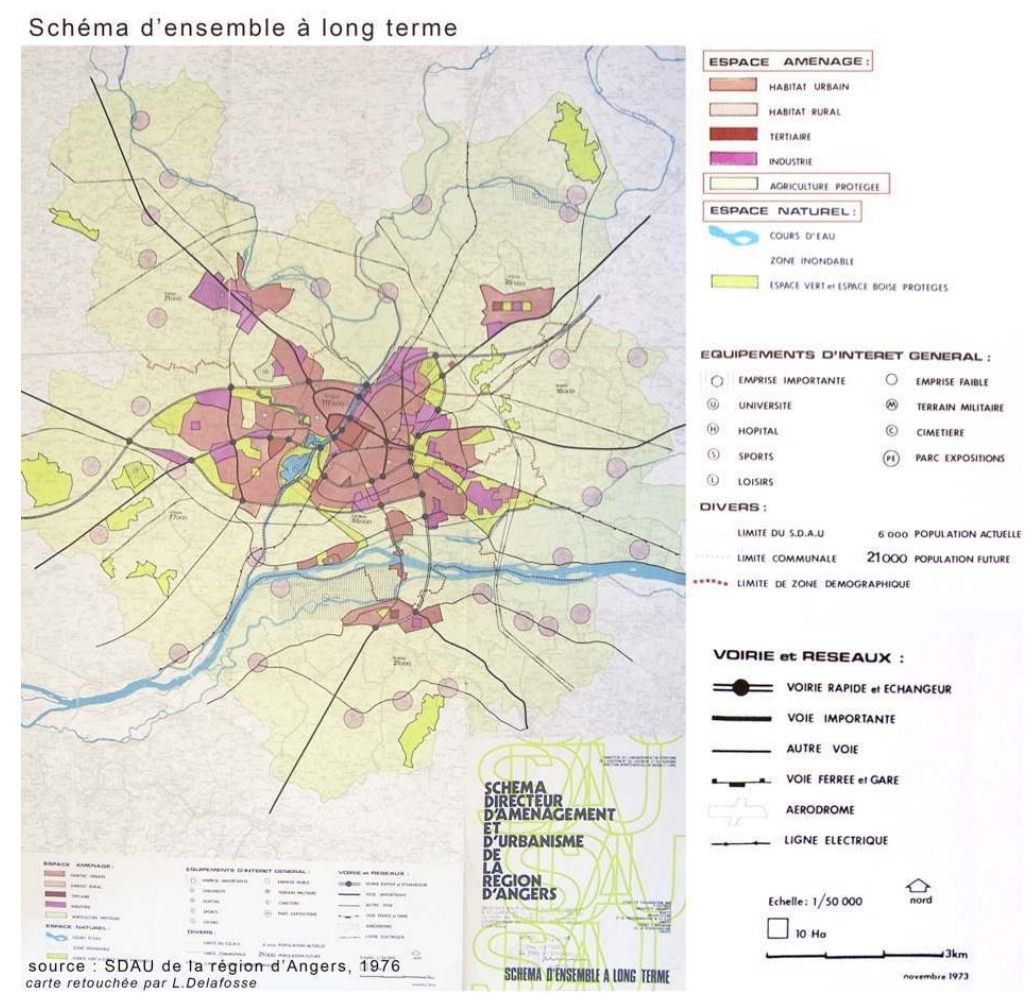

Fig. 3 : Des espaces libres envisagés sans continuité

11 L'agriculture, représentée en jaune sur le plan (fig. 3), est à protéger, non pas pour les paysages qu'elle façonne - à la différence du SDAURIF de la même époque - mais parce qu'elle est une activité productive performante, et en tant que telle, à développer ; l'accent est clairement mis sur son importance économique, en particulier celle des cultures spécialisées situées à l'est et au sud. À l'ouest, l'activité de polyculture élevage est passée sous silence, car elle ne rentre pas dans le projet de modernisation de l'époque ( ibidem: 51). Le paysage bocager qui en résulte, encore très présent à l'époque, est luimême ignoré car, outre le fait qu'il symbolise une agriculture "dépassée ", l'agriculture en soi n'est pas considérée comme productrice de paysage (Toublanc et Luginbühl, 2007 : 164 -165). De façon plus générale, la préoccupation paysagère exprimée dans le SDAU est centrée sur un objectif: lutter contre le "mitage " lié à l'urbanisation qui grignote le " cadre naturel» de la ville (ibid.: 52), i.e. des «sites de grande qualité $»^{5}$ (coteaux et crêtes surplombant les voies d'eau) perçus comme devant être protégés de la croissance économique et urbaine. 
Car, le projet est résolument économique. Outre la modernisation de l'agriculture, il promeut l'industrialisation de la région : extension portuaire, aménagement des « voies d'eau » - la Loire et la Maine - en "axes navigables », développement de voies express ( ibid. : 49). Coupant le centre-ville en deux, une voie rapide sera construite sur les berges de la Maine : elle témoigne d'une vision très éloignée de celle du projet actuel de trame verte et bleue.

Finalement, en 1976, les préoccupations écologiques sont réduites. Elles s'arrêtent à la reconnaissance des îles et des zones inondables pour le gibier d'eau et les oiseaux migrateurs (ibid. : 53). L'idée de continuité verte, absente des différents documents du SDAU, ne fonde pas le projet d'aménagement et d'urbanisme de la région d'Angers. En revanche, celui-ci véhicule une conception du vert articulée à une vision discontinue de la ville : une vision érigée encore aujourd'hui en principe, qu'il conviendrait de questionner.

14 À la différence du SDAU d'Angers, le SDAURIF approuvé en juillet 1976 met en avant les idées de trame verte (Estienne, 2010) et de ceinture verte à des fins récréatives, paysagères et/ou écologiques. Il affiche le leitmotiv de limiter spatialement l'urbanisation, et surtout de maintenir des discontinuités urbaines, mais à travers une nouvelle notion, celle de Zone Naturelle d'Equilibre (SDAURIF, 1976: 101; fig. 4). Le discours est très paysager ; le mot lui-même de paysage apparaît souvent, dans les titres notamment, ou dans les cartes, avec par exemple la notion de "zones d'ambiances paysagères " (ibidem:116). Il y est aussi beaucoup question de réseau et de composition pour les espaces ruraux mais aussi urbains. Le document affirme, et on est bien en 1976, «il reste beaucoup à faire pour parvenir à un véritable réseau organisé, desservant l'ensemble de la population. Il y a des espaces verts [urbains], il n'y a pas de réseau des espaces verts» (ibid. : 99). La politique de «trame verte» s'appuie sur deux principes: « un parti d'urbanisation discontinue le long des axes préférentiels et l'aménagement de coupures vertes d'intérêt général ou local; la définition et la protection d'espaces à dominante végétale combinées à la création d'un réseau d'espaces de loisirs adaptés aux besoins » (ibid. : 43).
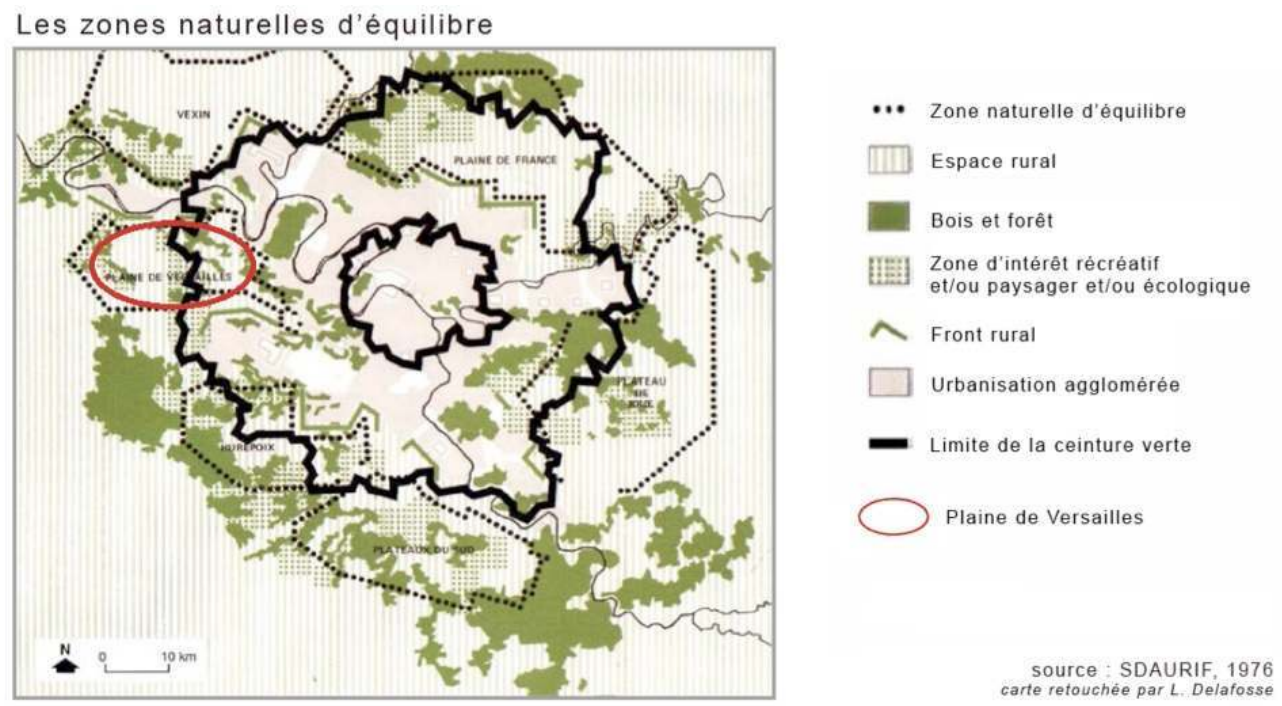
Fig. 4 : Une volonté d'inscrire les espaces libres dans un réseau de continuités

15 En cette décennie 1970, caractérisée par une critique de la croissance et de ses effets sur l'environnement, l'idée de trame verte est sous-tendue par des valeurs environnementales et paysagères émergentes : elle désigne un système d'espaces naturels reliés entre eux. Certes, il n'est pas encore question de corridors écologiques mais le front urbain est considéré comme devant être traité en maintenant un "glacis ", zone tampon entre la limite du bâti et la lisière des forêts, « nécessaire à la bonne gestion écologique de celles-ci » (SDAURIF, 1976:112). De même, une des orientations pour les zones naturelles d'équilibre est de "pratiquer une politique de restructuration foncière conciliant les nécessités de la production agricole, de la gestion des paysages et de la protection des équilibres biologiques ». Les promoteurs de ce schéma s'inscrivent dans la continuité des idées de Forestier qui déjà en son temps, tel un précurseur, avait porté une attention particulière aux espaces dédiés à (et produits par) l'élevage (modèle bucolique de la pastorale) et à l'activité forestière en les intégrant dans le parksystem sous l'intitulé "grandes réserves et paysages protégés (cf. supra). Ils iront cependant plus loin, en étendant la trame verte aux espaces agricoles cultivés, notamment en grandes cultures. Considérer l'agriculture, y compris intensive, comme une activité garante du maintien des espaces ruraux, l'envisager selon une vision dynamique articulant développement agricole et accueil des pratiques de loisirs constituent une approche inédite, avantgardiste et d'autant plus remarquable que l'agriculture et les espaces agricoles étaient absents du précédent schéma, le SDAURP' de 1965.

16 À l'évidence, le SDAURIF est un document fortement influencé par l'émulation intellectuelle qui a eu lieu au début des années 1970 autour du paysage. En effet, après un déclin dans la période de l'après-guerre, le paysage est redevenu, d'une manière peut-être plus intellectuelle qu'appliquée, un instrument au service du projet urbain. Dans le sillage de la création du ministère de l'environnement en 1971, plusieurs structures vont expérimenter et développer le paradigme du paysage d'aménagement : le Centre national d'études et de recherche sur le paysage, de 1972 à 1978 (Donadieu, 2009), les Organisations d'études et d'aménagement des aires métropolitaines (OREAM), de 1966 à 1983 (Pernet, 2011 : 415-418), l'École nationale supérieure du paysage créée en 1976. Quelques grands noms du paysagisme ${ }^{7}$ en sortiront. Ils revisitent les idées de cité-jardin ; ils proposent une mise en continuité entre ville et nature, avec le projet de «Métropole jardin » sur la Loire Moyenne (OREALM) qui défend une approche systémique : « un type particulier de conurbation constituée d'un chapelet de villes séparées et reliées par des espaces naturels aménagés le long du corridor fluvial» (Thibault et Verdelli, 2007) ayant une vocation récréative. Mais si une abondante littérature grise a été alors produite, elle a été peu publiée ou théorisée, et surtout elle n'a pas essaimé massivement dans les schémas directeurs qui resteront dominés par les approches économiques et fonctionnalistes.

17 L'exemple de la région Île-de-France montre finalement que l'idée de système vert se transmet au XXe s. dans le champ de l'urbanisme. Pourtant, même si « la notion de trame verte demeure largement débattue dans l'entre-deux-guerres» (Pousin, 2007), on n'en observe pas moins après la seconde guerre mondiale, un appauvrissement de la réflexion et en particulier du vocabulaire qui lui est associé. En outre, son aire de diffusion est restreinte à un cercle de professionnels éclairés, architectes et urbanistes, des visionnaires qui œuvrent plutôt dans des grandes villes. Autrement dit, cette idée héritée 
du passé ne se développe pas vraiment au-delà d'un groupe d'intellectuels initiés, proches de la haute fonction publique d'État; le passage de relais est assuré mais il ne fera pas progresser l'idée jusque dans les villes moyennes de province. Il faut dire aussi que les enjeux ne sont pas de même nature entre la région d'Angers et la Région Île-de-France. Cette dernière concentre une croissance démographique et un développement économique très importants. Dans un système politique centralisé, un déséquilibre s'est installé entre la capitale marquée par une dynamique d'urbanisation sans précédent et le reste du territoire (Gravier, 1947). Ce gigantisme urbain est à l'origine d'une mobilisation politique et intellectuelle qui a contribué à réactiver une idée ayant eu pour terreau le développement des grandes villes au XIXe siècle.

\section{2. À travers les schémas régionaux des années 1990, une montée des préoccupations paysagères et environnementales}

Après une éclipse partielle pendant les trente glorieuses, l'idée de trame verte revient en force à partir des années 1980 dans le champ de la planification et surtout, elle se répand massivement au-delà de la région parisienne. Elle est devenue un concept et un outil pour penser le développement urbain des villes, grandes et moyennes; en outre, elle apparaît comme une réponse aux préoccupations environnementales et paysagères qui animent les milieux de l'aménagement du territoire.

En témoigne le schéma directeur de la région angevine (SDRA) de 1996 qui mobilise explicitement la catégorie de "trame verte » en l'articulant à celle de paysage selon un double objectif urbanistique et social. Le terme générique « d'espace vert » présent dans le précédent schéma a laissé la place à un registre sémantique beaucoup plus riche et diversifié (cf. infra)qui reflète la vocation plurielle de la trame verte comme structure paysagère.

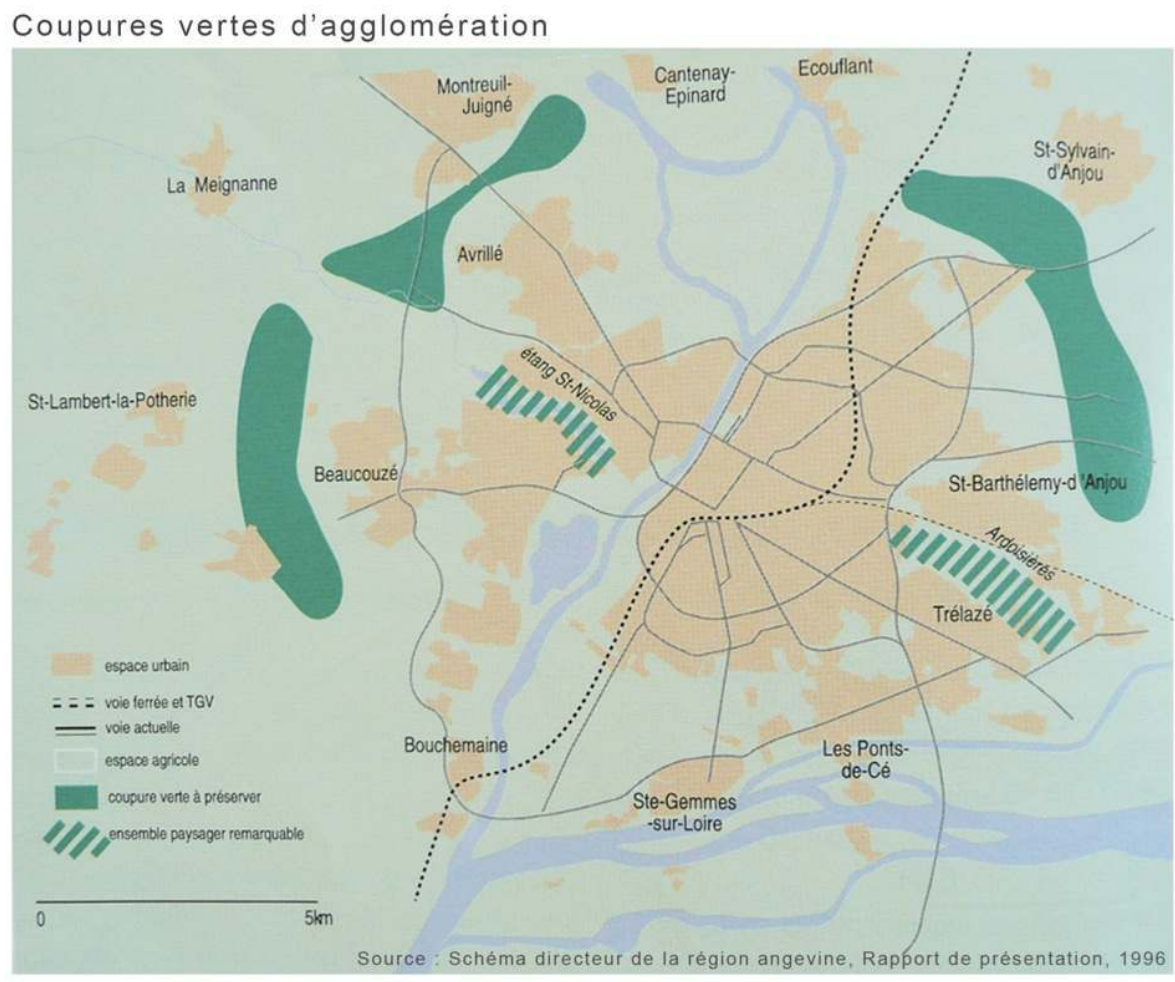


Fig. 5 : Esquisse inachevée de continuités vertes, ceinturant ou pénétrant l'agglomération de «maîtriser le front urbain » par des ruptures vertes, déjà présent dans les schémas précédents, est ici réaffirmé mais dorénavant rattaché à l'idée de continuité. Envisagées comme des maillons de la trame verte, trois "coupures vertes", également appelées " coupures d'urbanisation ou d'agglomération", sont identifiées : elles correspondent à l'ouest et au nord-ouest, à des espaces bocagers, à l'est, à des espaces périurbains hétérogènes, mêlant urbanisation, boisements, arboriculture fruitière et horticulture (fig. $\mathrm{n}^{\circ} 5$ ). Outre une représentation de la ville, contenue et discontinue, le principe de continuité verte sert une autre idée : ancrer la ville dans son territoire, l'arrimer à ses infrastructures "naturelles", à son socle géographique, la relier à la campagne qui l'environne. Le terme consacré est celui d'« armature paysagère ». Il s'agit de maintenir et de conforter « trois axes forts [ceux des vallées et du schiste] liés à la géomorphologie et à l'hydrographie qui structurent les paysages de l'agglomération» (SDRA: 62). Le raisonnement a pour postulat l'extension territoriale de l'idée de paysage, donc la continuité dans l'espace de ses composantes, qui ne limitent plus à quelques sites remarquables et circonscrits.L'accent est mis sur les interfaces ville/campagne et ville/ nature : des entre-deux ou des franges considérées par les urbanistes et les paysagistes comme des espaces de composition urbaine (Stefulesco, 1993).

En plus d'une fonction urbanistique, la trame verte (et plus largement le vert) sert également un objectif social : offrir des paysages quotidiens de qualité et des espaces de «nature » récréatifs, au cœur et à proximité de la ville, pour répondre aux attentes (ou présumées comme telles) des habitants. Dans le SDRA de 1996, il est formellement énoncé la nécessité d'assurer la continuité « des espaces naturels », notamment l'espace agricole et les boisements situés aux abords de la ville, de les protéger de la ville mais aussi pour la ville : afin de « garantir [leur] rôle futur d'espace de loisir périurbain » (SDRA : 167).

Dans un contexte marqué par une prise de conscience accrue des problèmes environnementaux, le SDRA affiche bien des thématiques comme la qualité de l'eau, les risques naturels et industriels, les déchets et les nuisances sonores. Mais contre toute attente, le projet de trame verte n'a pas pour ressort ces préoccupations, excepté la dernière qui est explicitement articulée à la « trame verte à traiter » (fig. $n^{\circ} 6$ ). Quant à la question de la richesse et du fonctionnement écologique, elle est peu évoquée dans le schéma, et surtout elle n'est pas clairement corrélée à l'idée d'une continuité verte, devenue pourtant un concept clé pour une discipline scientifique, nouvelle et montante, l'écologie du paysage ${ }^{8}$.

23 Les «éléments de nature » identifiés par les planificateurs et validés par les élus sont nombreux et beaucoup plus diversifiés que dans le SDAU de 1976. Le projet de trame verte consiste à renforcer ou à créer, et dans tous les cas, à articuler quatre catégories d'éléments très différents: «les plantations d'alignement [urbaines], le traitement paysager et phonique des nouvelles voies, les coupures vertes, le paysage bocager ». Contrairement au schéma de 1976, dans celui de 1996, les urbanistes font la part belle aux paysages agricoles à caractère bocager situés à l'ouest en considérant qu'ils sont à "conforter ou à reconstituer». Ces territoires façonnés par de la polyculture élevage les intéressent non pas d'un point de vue économique mais d'un point du vue paysager. Dans le même temps, les paysages agricoles produits par les cultures spécialisées, horticulture et arboriculture à l'est et viticulture au sud, ne sont plus mis en avant dans le schéma vert 
9. Or, le bocage, démantelé par l'évolution de l'agriculture, est beaucoup moins présent sur le territoire angevin en 1996 qu'en 1976 : c'est sans doute parce qu'il est en train de disparaître que les planificateurs sensibles à sa régression lui redonnent un droit de cité comme élément essentiel du projet de trame verte. En l'espace de 20 ans, le regard sur les haies a basculé : d'abord ignorées voire dévalorisées car synonymes d'archaïsme, elles sont dorénavant perçues comme des éléments de nature (Delbos et Jorion, 1988 : 17-18; Toublanc et Luginbühl, 2007, op. cit.).

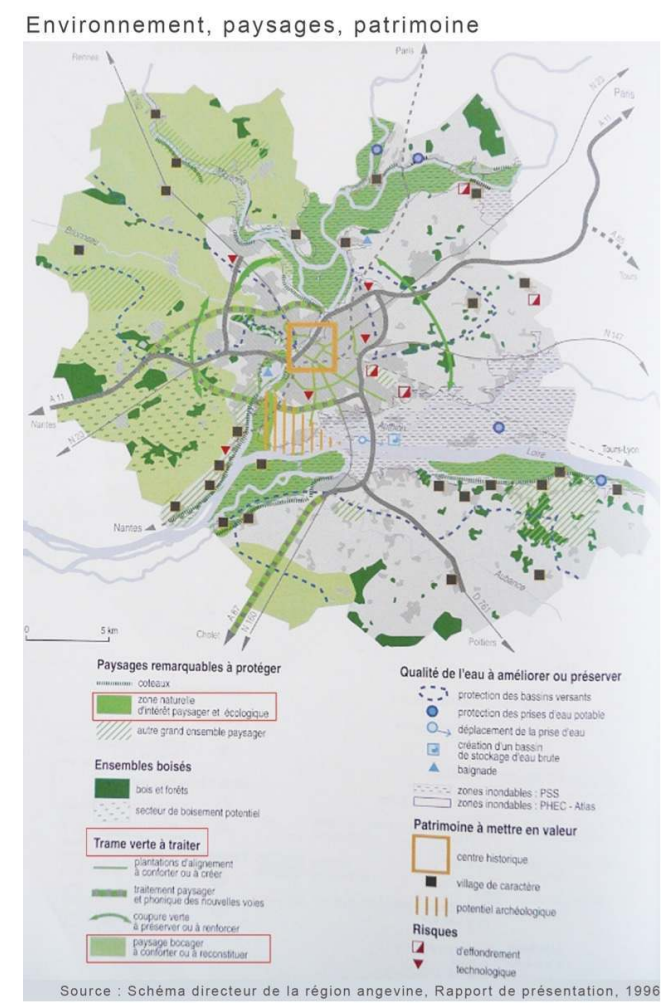

Fig. 6 : Une trame verte prenant appui sur le bocage

Le revirement face au bocage, entre les deux schémas de 1976 et 1996, ne semble pas avoir pour fondement explicite une reconnaissance de sa valeur écologique émergente. D'abord le bocage n'entre pas dans la catégorie "espaces naturels sensibles» (SDRA : 152-153). Seuls deux types d'espaces sont conçus comme tels:les vallées inondables (principalement la Loire et les Basses vallées Angevines ${ }^{10}$ ) et les boisements, au demeurant peu nombreux. Les premières, dénommées «zones naturelles d'intérêt paysager et écologique ", occupent une place de choix. Les seconds, considérés comme des écosystèmes intéressants, - certains étant d'ailleurs déjà référencés comme ZNIEFF -, sont placés au cœur d'un projet de « réseau d'espaces forestiers paysans » (idem: 198). Par ailleurs, dans le rapport de présentation, il n'y a aucun indice sur une participation éventuelle d'écologues du paysage à la fabrication du SDRA, pas même une référence bibliographique à leurs travaux. Cela n'a sans doute rien de surprenant car ceux-ci ont participé dés les années 1980 à titre d'experts aux actions d'aménagement rural (remembrements fonciers, aménagements hydrauliques) pour lesquels la loi de 1976 sur la protection de la nature exige la réalisation d'études d'impact sur l'environnement (Baudry et Burel, 1999). Mais, il faudra attendre la décennie 2000 pour qu'ils élargissent leur champ d'action et investissent la planification urbaine (Descamps, 2004 ; Clergeau, 2007). Pour autant, les recherches conduites par l'écologie du paysage ont très 
certainement influencé le schéma de 1996 de la région angevine en modifiant indirectement le regard des planificateurs sur le bocage. En effet, en choisissant le bocage comme objet de recherche privilégié, cette discipline a contribué à sa reconnaissance, audelà même du champ scientifique.

La survalorisation d'une structure géographique, le bocage, dont les raisons d'être et les fonctions passées sont aujourd'hui révolues, alimente un hiatus entre une logique sociale et économique et une logique de reconnaissance paysagère ${ }^{11}$. Elle témoigne cependant de ce que progressivement, le territoire rural n'est plus seulement pour les planificateurs une coupure à préserver dans le tissu urbain, mais un réseau ayant d'autres valeurs, notamment paysagères.

Dans le SDRA, l'identification de la continuité verte s'appuie d'ailleurs sur une expertise avérée des paysages (Cros et al : 1993). Cela reflète de façon indiscutable la montée en puissance des préoccupations paysagères dans la société. Le renouveau de la pensée du paysage, qui a marqué la décennie 1970, s'est poursuivi, relayé par le Ministère de l'Environnement et du Cadre de Vie et celui de l'Agriculture, - à travers respectivement la «Mission du paysage» et le bureau "Cadre de vie, environnement et paysage " (Toublanc, 1989: 51 - 88). Au cours des années 1980, ces structures étatiques affirment que le paysage est partout, qu'il est pluriel et vivant. «Il n'y a pas de petits paysages » (Ricquois $^{12}, 1983: 57$ ), il y a des "paysages quotidiens, [...] qui ne justifient à priori ni protection exceptionnelle, ni création forte $[. .$.$] où pourtant vivent et travaillent une$ majorité de gens » (Ricquois, 1983: 56). Cela conduira notamment à la publication de la Loi Paysage (1993) qui élargit l'idée de paysage à celle de cadre de vie et les politiques paysagères aux territoires ordinaires. Participant au même mouvement, dès la fin des années 1980, se développe une dynamique de recherche importante sur ce thème.

Si le SDRA de 1996 est en accord avec la vision paysagère de l'époqueet ne peut pas être décrypté sans se référer à ce contexte, il n'en porte pas moins l'empreinte, ne serait-ce que par ricochet, de la montée des préoccupations environnementales. En effet, pour certains auteurs, il existe un lien entre l'importance accordée aux questions de paysage et l'attention portée aux questions environnementales, les secondes étant le ferment des premières ${ }^{13}$. «La chance du paysage tient aujourd'hui à [...] une sensibilité sans cesse accrue du public aux problèmes d'environnement » (Pigeat, 1981:3). Il faudra cependant attendre la fin du $\mathrm{XX}^{\mathrm{e}} \mathrm{s}$. pour que l'idée de continuité verte soit associée directement aux préoccupations écologiques.

\section{3. Écologie et paysage : deux approches qui deviennent autonomes?}

L'appropriation de l'idée de continuité verte par l'écologie du paysage dans un autre registre, celui des processus biologiques, joue un rôle certain dans le regain d'intérêt pour ce paradigme à la fin de la décennie 1990. Véhiculé par une discipline scientifique ayant prouvé sa capacité à s'emparer d'une question de société, la protection de la nature en milieu urbain (Clergeau, op.cit.), la continuité redevient un moteur de projet dans les champs de l'aménagement du territoire et de la planification urbaine; deux domaines empreints de l'idéologie du développement durable. Quelques années plus tard, à la faveur du Grenelle de l'Environnement (2007) qui met l'accent sur l'érosion de la 
biodiversité, l'idée est reprise et amplifiée à travers l'expression consacrée de «trame verte et bleue ».

Le SCoT (Schéma de Cohérence Territoriale) du Pays Loire Angers et le projet de planification de la Plaine de Versailles donnent à voir la façon dont l'idée est réactivée et réinterprétée par les praticiens et les élus en charge de l'élaboration des documents d'aménagement sur ces deux territoires, à une époque où développement durable rime avec biodiversité et où l'un et l'autre sont devenus incontournables. En effet, si l'on s'en tient au discours, l'idéologie du développement durable codifié dans de nombreuses lois (ex. loi SRU de 2000, loi Voynet de 1999) va surdéterminer l'action publique et entraîner dans son sillage celle de réseau vert.

\subsection{La continuité verte dans la planification de l'agglomération angevine : deux lectures parallèles, écologique et paysagère.}

Le SCoT du Pays Loire Angers concerne 66 communes, 300000 habitants, une communauté d'agglomération et 3 communautés de communes; il a été mis à l'étude en 2005 et adopté le 21 novembre 2011. Dans le SCoT de 2011, l'idée de continuité verte, articulée à celle du développement durable, est mise en avant bien plus encore que dans le SDRA de 1996. La terminologie s'est encore enrichie, elle met dorénavant l'accent sur la mise en relation: outre les formulations utilisées jusqu'alors, d'autres voient le jour comme "liaisons naturelles», " pénétrantes vertes", "continuités écologiques", « continuités paysagères ", « corridors humides », " corridors verts », etc.Ces expressions renvoient à deux projets différents, déclinés à l'échelle de la région angevine et de chaque polarité urbaine : l'«armature verte et bleue » et la "trame écologique verte et bleue ». Le premier s'inscrit dans une vision paysagère (fig. 7), le second dans une vision écologique (fig. 8).

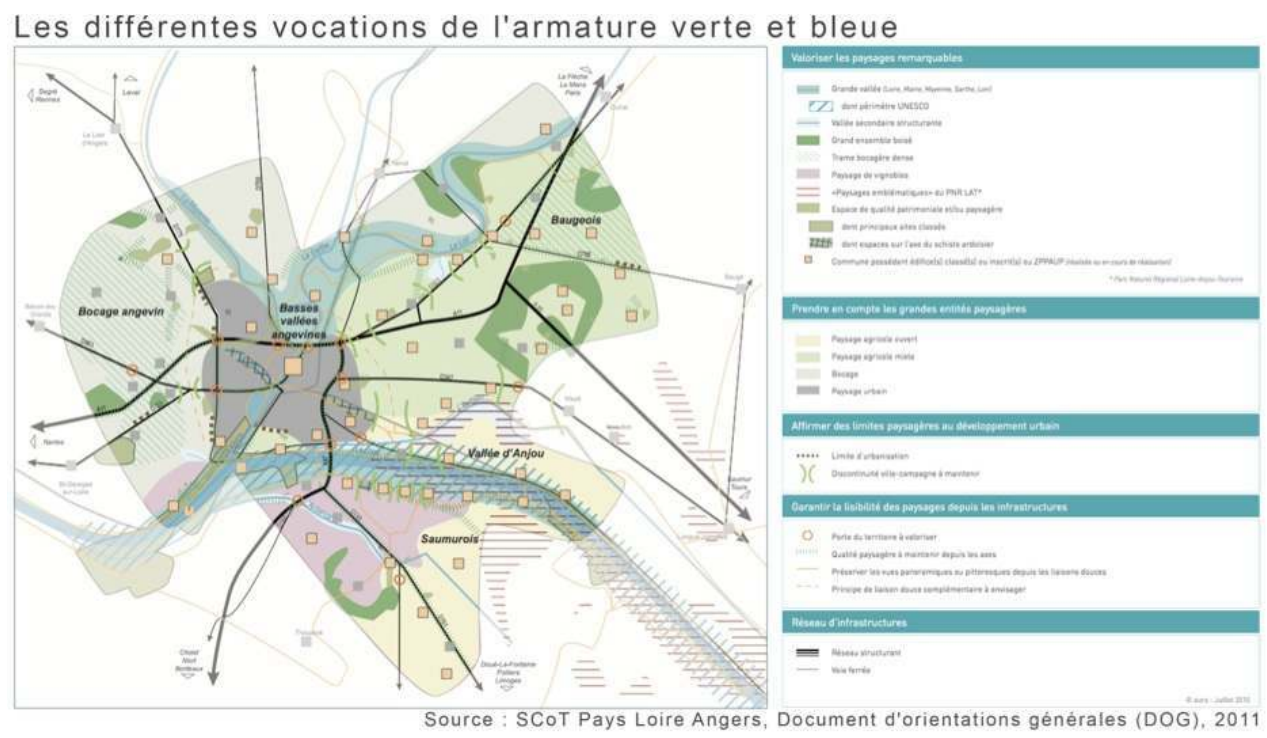

Fig. 7 : La prise en compte de l'ensemble des espaces par l'approche paysagère

«L'armature verte, c'est ce qui reste une fois qu'on a retiré les espaces urbanisés... soit 85 $\%$ du territoire » (chargé de mission, Agence d'urbanisme de la région d'Angers/AURA, 2009). L'expression désigne une grande diversité d'espaces, exceptionnels ou ordinaires, 
productifs (agricoles et forestiers principalement) ou non (vallées inondables, anciennes ardoisière). Du micro-local au territoire plus vaste, c'est la matrice territoriale et le cadre paysager dans lesquels la ville s'est développée, mais ce sont aussi les structures et les composantes végétales urbaines. La nécessité de raccorder les espaces constituant l'armature verte et bleue, est ainsi affirmée dans le SCOT : « valoriser le réseau de liaisons naturelles et semi-naturelles » (Projet d'aménagement et de développement durable/ PADD, 2010 : 59)

La mise en réseau vise à « organiser le développement des territoires »; c'est un outil de planification au service d'une structuration multipolaire de la ville. Pour lutter contre un continuum urbain, deux idées fortes sont revendiquées : d'abord, assurer une continuité entre les espaces de nature en ville et l'espace rural périphérique en «renforçant les pénétrantes vertes et en les reliant aux espaces de campagne». Ensuite, il s'agit de «maintenir une interface [...] non construite » (ibidem : 59) autour du pôle métropolitain et entre les sept « polarités » de développement urbain afin d'en « renforcer l'identité», et "d'améliorer les entrées d'agglomération " (ibid. : 54). Pour ce faire, deux orientations sont privilégiées : " préserver les espaces agricoles » et «affirmer des limites paysagères fortes » aux espaces urbanisés (Document d'orientations générales/DOG, $2010: 69-72$ ). À travers cette fonction d'exosquelette, on retrouve l'idée des coupures ou transitions vertes visant à maintenir des « discontinuités ville-campagne ».

Cependant, l'armature verte n'a pas qu'une vocation urbanistique. Développement durable oblige, l'idée de continuité verte est repensée par les acteurs en charge du SCoT à l'aune des grands principes de cette utopie; elle est construite pour répondre aux trois piliers - social, économique et écologique, et elle se voit par conséquent attribuer une multiplicité de fonctions concomitantes. Il est affirmé «l'opportunité d'un réseau vert aux multiples usages ». Les objectifs sociaux peuvent être résumés ainsi : maintenir des espaces d'aménité et des paysages caractéristiques concourant à l'image du territoire, permettre l'accès à la nature et aux rivières pour tous les habitants de la métropole, leur offrir un cadre de vie attractif et des paysages quotidiens de qualité (fig. 7). C'est le paysage perçu et vécu par les habitants. Ensuite, il y a des objectifs économiques productions agricoles et forestières, activités touristiques, ressources énergétiques - que les espaces reliés entre eux sont supposés remplir.

En revanche, les objectifs écologiques ne sont pas portés par l'armature verte. En référence à l'un des objectifs du Grenelle de l'Environnement (2007) ${ }^{14}$ - la mise en place au niveau national « d'une trame verte complétée d'une trame bleue »-, le SCoT affiche un projet de«réseau écologique» pour «valoriser le maintien de la biodiversité » (fig. 8). Cette finalité première, énoncée de façon précise et circonscrite, n'en reste pas moins noyée dans un discours passant en revue les thématiques sociales, économiques et environnementales qui structurent l'idéologie du développement durable. 


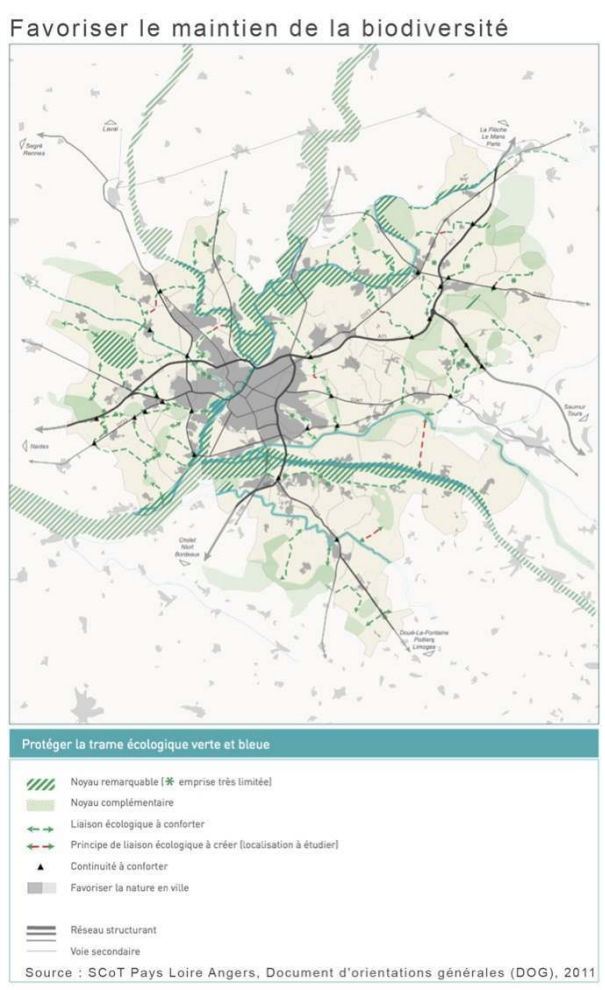

Fig. 8 : La définition d'un réseau linéaire par l'approche écologique «l'armature verte et bleue » qui produit du paysage et «la trame écologique verte et bleue » qui produit de la biodiversité. L'exemple type est celui des territoires viticoles au rôle paysager pleinement reconnu mais dont la fonction écologique, compte tenu des pratiques de production, pose question. Désignant des espaces repérés pour leur valeur écologique, la trame a une emprise spatiale beaucoup plus restreinte que l'armature verte dont elle n'est qu'une composante et dans laquelle elle est emboîtée. La nature des savoirs et des connaissances mobilisés pour l'identification de l'une et de l'autre est fondamentalement différente. L'identification de la trame a requis une expertise inédite et très approfondie, naturaliste et écologique, mobilisant à la fois le milieu associatif (association "Études des Équilibres Naturels»), la recherche (Conservatoire national botanique de Brest), et un bureau d'études privé regroupant des compétences écologiques diverses. En revanche, la définition de l'armature n'a pas conduit à une expertise paysagère nouvelle ${ }^{15}$. Elle s'est appuyée sur des études déjà anciennes (Atlas des Paysages du Maine-et-Loire, 2003 ; Z. Cros et al, 1993 cf. supra).

Les éléments et les milieux qui composent la trame sont divers : de la vallée inondable perçue comme une trame verte territoriale à l'échelle du SCoT, à la mare, élément ponctuel, identifiable au niveau de la parcelle. Leur identification s'appuie sur la théorie et le vocabulaire de l'écologie du paysage (noyaux, corridors, matrices) ${ }^{16}$; ils sont hiérarchisés selon leur richesse et leur rôle biologique en deux réseaux, « remarquables et complémentaires ». Le premier relie des habitats déjà reconnus (ZNIEFF 1, stations d'espèces rares), bien souvent protégés (Natura 2000, site RAMSAR, mesures agroenvironnementales); le second correspond à des espaces plus ordinaires sur lesquels il n'existe pas et il n'est pas prévu de prescriptions fortes. En fin de compte, le projet de 
«trame écologique verte et bleue» semble entériner une situation existante, et in fine renforcer l'attention portée aux espèces patrimoniales.

Cette hiérarchisation est accentuée par les intentions exprimées dans le SCoT : la trame a vocation à être "protégée ", l'armature à être simplement «consolidée et valorisée " (DOG, 2010 : 16), car sa gestion échappe à la puissance publique du fait notamment du statut privé des espaces concernés et de leur fonction, le plus souvent productive. Une situation qui n'est pas sans rappeler la création des parcs nationaux en France avec comme principe celui d'une partition entre des réserves naturelles intégrales (zone centrale) et des zones périphériques ouvertes à l'activité humaine.

En définitive, on ne peut que s'interroger sur les effets d'une telle distinction entre trame verte et armature verte. Outre qu'elle conduit à une division du territoire, elle renvoie à une pratique, celle du zonage, très répandue dans la planification urbaine depuis l'aprèsguerre qui consiste à protéger les espaces exceptionnels au détriment du reste du territoire, laissé pour compte et livré sans régulation publique, aux processus économiques.

\subsection{Une situation de région parisienne : la plaine de Versailles. Un jeu d'échelles inversé entre paysage et écologie, entre l'échelon régional et local.}

39 Dans certains projets de planification (situation angevine), le paysage apparaît comme dissocié de l'environnement, dans d'autres, il est considéré comme une alternative opportune à une planification environnementale, quand d'autres enjeux locaux, notamment agricoles, s'imposent. Tel est le cas de la plaine de Versailles. Celle-ci était désignée comme zone naturelle d'équilibre dans le SDAURIF de 1976 ( $c f$. supra fig. 4), selon un périmètre proche de ce qui est devenu le champ d'action de l'association patrimoniale de la Plaine de Versailles et du Plateau des Alluets, créée en 2004 (Pupin, 2008). Elle appartient, dans son tiers oriental, proche du parc du château de Versailles, à la ceinture verte de la région Ile-de-France (SDRIF de 1993; voir Brédif, Pupin, 2012). Mais il est surtout intéressant de voir son statut évoluer aujourd'hui comme " espace ouvert ", en cohérence avec la conception des zones naturelles d'équilibre qui tendait à reconnaître des caractères similaires aux champs, aux forêts, aux espaces verts; comme " pénétrante agricole» (SDRIF 2008); et finalement, comme territoire agri-urbain. Son extrémité ouest est aujourd'hui concernée par une "continuité écologique majeure ", pièce d'un arc francilien dans le SDRIF de 2008. Ce document régional n'utilise pratiquement pas le paradigme du paysage; mais il énonce des principes conformes à ce qui est développé à la fois dans les lois du Grenelle de l'environnement, en matière de trames vertes à différentes échelles, et par l'écologie du paysage, à savoir la recherche de connexions biologiques. Ainsi est proposé un système régional d'espaces ouverts, confondant dans une même vocation de préservation et de mise en réseau, « les espaces agricoles, boisés et naturels ». L'espace agricole fait l'objet d'un développement particulier, reprenant des fonctions déjà exposées dans le schéma de 1976, mais y ajoutant des fonctions environnementales plus précises (eau, biodiversité, sol, risques, notamment), et une fonction d'adaptation au changement climatique. L'application du principe de mise en connexion biologique des espaces sur ce territoire, situé hors PNR, est difficile car les acteurs locaux sont face à un certain dénuement en matière de leviers réglementaires intercommunaux. D'ailleurs, c'est un territoire actuellement sans SCoT, 
sans SDAU(le dernier, datant de 1983, est caduc; un SCoT est en cours d'approbation sur une partie du territoire de l'association). Un certain nombre de projets ont cependant émergé, sur ces principes écologiques, comme le développement des bandes enherbées, ou la renaturalisation du ru de Gally, principal cours d'eau, quoique modeste, de cette petite région. Ces initiatives sont considérées par les agriculteurs comme des menaces pour leur activité. L'association patrimoniale, qui finalement se présente comme une instance de gouvernance interterritoriale, héritière du périmètre de la zone naturelle d'équilibre, a notamment pour objectif de prendre en compte les préoccupations de ces agriculteurs. Il est intéressant de constater que c'est dans l'outil "paysage » que l'association cherche actuellement des solutions pour concilier l'identité agricole et la mise en œuvre des principes écologiques : il ne s'agit plus du paysagisme du XIXe siècle, centré sur la création de parcs urbains et sur l'aménagement des villes, mais d'un paysagisme de concertation et de médiation, de fédération des différents acteurs. Ainsi, l'association, à l'initiative du collège des agriculteurs, est dans une démarche d'élaboration d'une charte paysagère, avec pour objectif de l'annexer aux futurs SCoT du territoire. Cette charte vise d'abord à préserver une cohérence paysagère autour de l'entité plaine de Versailles, par-delà les découpages administratifs.Elle cherche aussi à intégrer les principes de connectivité biologique au développement social et économique, notamment agricole.

40 Le cahier des charges de cette charte paysagère a été élaboré par l'association à partir d'un diagnostic des paysages qui a proposé parmi les actions prioritaires une mise en réseau des continuités écologiques porteuses d'usages. Ces orientations ont ensuite fait l'objet d'une enquête auprès des acteurs locaux, pour aboutir à la formulation d'un objectif : « faire de ces paysages des lieux de rencontres et d'échanges entre les différents acteurs afin d'envisager collectivement l'aménagement des espaces qu'ils partagent » (version du 18 novembre 2011) ; la co-construction des orientations avec les acteurs locaux revient comme un leitmotiv. L'idée de trame verte a donc surtout servi de mobilisation locale et de prise de conscience de la valeur paysagère de l'espace agricole ; l'avenir de la charte paysagère nous dira ce qu'il en est de la prise en compte des processus biologiques.

41 On retrouve dans ce cas de figure une situation proche de celle des PNRfranciliens, qui connaissent aussi des difficultés pour mettre en œuvre des projets de trame verte: une enquête auprès des chargés de mission des parcs a montré combien le paysage constitue localement un levier d'action important (Guerraoui, 2011). Le recours au paysage et les outils utilisés (visite de terrain, lecture in situ des paysages commentée par un expert écologue, mise en images, iconographie paysagère, ...) permettent une traduction des principes de la préservation des continuités écologiques, plus facilement saisissable localement. Compréhension de l'espace plus que connaissance des milieux, l'approche paysagère est plus facilement appropriable, en même temps qu'elle opère aussi une simplification qui complète mais aussi oriente les stratégies d'action. L'approche écologique est alors conçue comme nécessaire en tant qu'elle donnera aux enjeux paysagers une valeur fonctionnelle jugée positivement.

42 Le SCoT, le SDRIF et le projet de charte paysagère sur la Plaine de Versailles sont le reflet $\mathrm{du}$ renouvellement et de la diffusion de la pensée du paysage, depuis maintenant plusieurs décennies, dans le champ de l'aménagement du territoire comme dans celui de la recherche, en particulier les sciences de l'homme et de la société qui depuis le milieu des années 1990 se sont emparées de cette question. Ce mouvement, dont le point d'orgue 
est sans doute la Convention européenne du paysage de 2000 ratifiée par la France en 2006, fait la part belle aux composantes sociales et historiques des paysages, à la participation des populations, à leurs perceptions et à leurs façons de qualifier leurs paysages quotidiens ; il se pose d'ailleurs parfois tel un courant d'opposition, ou du moins telle une vision critique, face au « tout écologie » qui émerge alors (Roger, 1997). Montée en puissance de la prise en compte paysage d'un côté, de l'écologie de l'autre, les relations entre les projets de trame verte et les enjeux paysagers n'en demeurent pas moins ambivalentes.

\section{Conclusion}

Après un âge d'or paysager, puis une éclipse partielle, l'idée de continuité verte revient au cours des années 1980 - 1990 dans la planification urbaine à la faveur d'un renouveau de la pensée du paysage et d'une montée des préoccupations environnementales. Dés la fin des années 1990, elle est enserrée dans l'idée de développement durable et au cœur d'un débat sur la multifonctionnalité. Aujourd'hui, elle est prise en étau entre planification écologique et planification urbaine ; une tension qui conduit à méconnaître les fonctions sociales, économiques et paysagère des espaces de nature et à penser la biodiversité, ordinaire et exceptionnelle, comme un enjeu exclusif. Si le Grenelle de l'Environnement a fait renaître sous de nouveaux auspices l'idée fort ancienne de continuité verte, et l'a mise au service d'un objectif en apparence bien circonscrit, la conservation des espèces, il a conduit à séparer paysage et environnement. Des considérations écologiques complexes (la biodiversité, le climat), et surtout de dimension planétaire, ne relaient plus les préoccupations paysagères (Emelianoff, 2001; Fabiani, 2001). Le paysage arrive en sixième et dernière position quant aux finalités des trames vertes, telles qu'exprimées dans la "loi portant engagement national pour l'environnement" (dite Grenelle 2, loi 2010-788 du 12 juillet 2010, article 121). Les écologues du paysage eux-mêmes revendiquent - en dépit de la dénomination de leur discipline - qu'ils n'ont pas vocation à porter les enjeux paysagers tels qu'ils sont aujourd'hui compris dans l'aménagement du territoire (Baudry, $2010: 29-55)$. Certaines situations, sans doute exceptionnelles, comme celle de la Plaine de Versailles, montrent au contraire que le paysage, à un échelon local, peut aussi servir à faire accepter des objectifs écologiques et à les intégrer dans des enjeux socio-économiques, redevenant ainsi un outil de planification à part entière. C'est aussi par le biais de la participation devenue un principe incontournable que le paysage ressurgit ; pour ainsi dire, par obligation.

\section{BIBLIOGRAPHIE}

Baudry J. \& al., 2010, « Comment rapprocher l'écologie du paysage et le projet de paysage ? », Les Carnets du Paysage, n¹9, p. 20-55.

Baudry J. \& Burel F., 1999, Écologie du paysage. Concepts, méthodes et applications, Tec \& Doc, Paris, $359 \mathrm{p}$.

Développement durable et territoires, Vol. 3, n² 2 | Juillet 2012 
Baudry J. (dir.), 2009, Trames vertes, rapport de synthèse, programme de recherche « Paysage \& Développement Durable ", 160 p.

Brédif H., Pupin, V., 2012, « Réévaluer la place de l'agriculture à l'heure du Grand Paris », Annales de Géographie, 683, p. 43-65.

Clergeau P., 2007, Une écologie du paysage urbain. Apogée Ed., 140 p.

Cormier L. et al., 2010, « La planification des trames vertes, du global au local : réalités et limites ", Cybergéo: European Journal of Geography, 22 p.

Cros Z., Luginbühl Y., Croneau R., Toublanc M., 1993, Schéma directeur de la région angevine. Étude paysagère, DDE 49/SEGESA/CNRS, $67 \mathrm{p}$.

Décamps H. \& Décamps O., 2004, Au printemps des paysages, Paris, Buchet-Chastel, 232 p.

Décarie J., 2002. « Le réseau vert « système sympathique » de la ville post industrielle », in :

Malizieux J., Sénécal G., Manzagol C. (dir.), Grands projets urbains et requalification, Québec, Presses Universitaires du Québec, pp. $202-213$.

Delbos G. \& Jorion P., 1988, « La Nature ou le réel forclos », in Chasser le naturel..., Cadoret A. (dir.), Cahier des Études rurales, 5, Paris, EHESS, p. 17-18.

Donadieu P., 2009, «Eléments pour une histoire de la recherche à l’Ecole Nationale Supérieure du Paysage », Projet de paysage, http://www.projetsdepaysage.fr/fr/

elements_pour_une_histoire_de_la_recherche_a_l'ecole_nationale_superieure_du_paysage_de_versailles_ensp_

Emelianoff C., 2001, « Un nouveau modèle urbain », in L'environnement, question sociale (collectif), Paris, Odile Jacob, p. 292-290.

Estienne I., 2010, L'intervention du paysagiste dans la ville, de 1960 à aujourd'hui : pertinence et enjeux pour les architectes et les urbanistes. Le cas de la métropole Lilloise, Thèse de l'Université de Lille 1, 441 p.

Fabiani J.-L., 2001, « L'amour de la nature », in L'environnement, question sociale (collectif), Paris, Odile Jacob, p. 39-47.

Fabos J.-G., 2004. "Greenway planning in the United States : its origins and recent case studies". Landscape and Urban planning, 68, p. 321-342.

Fabos J.-G., Ahern J., (eds.), 1995, Greenways : the beginning of an international movment. Amsterdam, Elsevier, $498 \mathrm{p}$.

Forestier J. C. N., 1908, réed. 2001, Grandes villes et systèmes de parcs, Paris, Norma, 384 p.

Guerraoui S., 2011, Faire des paysages l'outil d'une politique d'aménagement partenariale : l'exemple des territoires ruraux d'Île-de-France dans la perspective de mise en cuvre du SDRIF 2008, Mémoire de Master 2 DYNATERP Paysages et médiations, Université d'Angers/AgrocampusOuest/EPICEA Développement/Région Ile-de-France, $100 \mathrm{p}$.

Harter H., 2002. «F. Law Olmsted ou l'art paysager américain », Urbanisme, n³25, p.79-84.

Le Dantec J.-P., 1996, Jardins et paysages, Paris, Larousse, p. 361-368.

Leveau T., 1964, «Les espaces verts dans la cité future », Espaces verts, n¹, décembre 1964, p.13-21.

Luginbühl Y., Toublanc M., 2003, «L'évolution des représentations collectives et des modes de gestion du bocage ", in : J.Baudry, A. Jouin (dir.), De la haie aux bocages. Organisation, dynamique et gestion, Versailles, INRA-Editions, p. 43-73. 
Maumi, C., 2009. Usonia ou le mythe de la ville-nature américaine, Paris, Éditions de la Villette, 240 p.

Ménard P., Clergeau P., 2001, « La notion de zone de connexion biologique : son application en aménagement du territoire ", Mappemonde, $\mathrm{n}^{\circ}$ 64, p.24- 29.

Moindrot, C., 1961, « Un essai de planification du paysage. Les zones vertes des villes britanniques ", Annales de géographie, t.70, n³82, p. 585-596.

Oberdoerffer M. P., 1964, « Le plan vert de Paris », Espaces verts, n¹, décembre 1964, p.20-22.

Pernet A., 2011, Le grand paysage en projet, entre trajectoires institutionnelles et territoire vécu, Thèse de l'Université Paris 1, 620 p.

Pigeat J.-P., 1981, Paysages, catalogue d'exposition Centre Georges Pompidou, 96 p.

Planning Policy Guidance 2 : Green Belts, Department for Communities and Local Government, http:// www.communities.gov.uk/documents/planningandbuilding/pdf/155499.pdf

Pousin F., 2007, « Du townscape au paysage urbain, circulation d'un modèle rhétorique mobilisateur », STRATES (13), CNRS, p. 25-50.

Pupin V., Viel J.-M., Colin M., 2008, « Une démarche patrimoniale de gestion de la qualité du vivant en Ile-de-France : l'expérience de la plaine de Versailles et le Plateau des Alluets ", Le Courrier de l'Environnement de l'INRA (55), p. 125-134.

Rabreau D., 1991, «La promenade urbaine en France au XVII ${ }^{\mathrm{e}}$ et XVIII ${ }^{\mathrm{e}}$ s. : entre planification et imaginaire ", in : Mosser M., Teyssot G., Histoire des jardins de la Renaissance à nos jours, Paris, Flammarion, p.301-312.

Racine M. (dir.), 2002, Créateurs de jardins et de paysages en France du XIXe au XXIe siècle, Arles, Actes Sud/ENSP, $421 \mathrm{p}$.

Rambaud I. (dir.), 2009, Patrimoine et paysages, Conseil général de Seine-et-Marne, éd. Lieux-dits, $224 \mathrm{p}$.

Ricquois A. (interviewé par Véronique Granger), 1983, Le paysage au quotidien, Métropolis, Paris, 58/59, p. 55-62.

Roger A., 1997, Court traité du paysage, Gallimard, 199 p.

Santini C., 2011, " "Les promenades de Paris" de Charles-Adolphe Alphand », Projets de paysage, http://www.projetsdepaysage.fr/fr/_les_promenades_de_paris_de_charles_adolphe_alphand_

Stefulesco C., 1993, L'urbanisme végétal, Paris, Institut pour le développement forestier, 323 p.

Thibault S., Verdelli L., 2007, « La métropole jardin, un projet urbain régional jamais complètement abandonné », Loire et ses terroirs, n60, 2007, p.19-24.

Toublanc M., 1989, L'état et les espaces abandonnés. L'exemple de la terrasse méditerranéenne, Thèse de doctorat, EHESS, Paris, $335 \mathrm{p}$.

Toublanc M., Cormier L., Baudry J., Guttinger P., Lizet B., 2008, « The "trame verte" in France : history and movement of a concept », 23rd Session of PECSRL «Landscapes, Identities and Development », Lisbon/óbidos, Portugal, 1-5 Septembre 2008.

Toublanc M., Luginbühl Y., 2007, « Des talus arborés aux haies bocagères : des dynamiques de pensée du paysage inspiratrices de politiques publiques », in : Berlan-Darqué M., Luginbühl Y., Terrasson D. (dir.), Paysages : de la connaissance à l'action, Montpellier, Éditions Quae, p.163-177. 


\section{NOTES}

1. 31 communes, 51000 ha, 283000 habitants en 2008 , constituant un bassin de vie cohérent.

2. 24 communes, 18000 ha, 126000 habitants en 2008 , aux marges de différentes agglomérations.

3. La promenade plantée est une spécificité de l'urbanisme français qui se développe au cours des $17 \mathrm{e}$ et $18 \mathrm{e}$ siècles. Elle renvoie à des pratiques sociales de la bourgeoisie comme : se promener en ville ;avoir des vues sur la campagne depuis la ville ;voir la ville depuis des promontoires aménagés, en somme voir et être vu (Rabreau, 1991).

4. Le terme région est employé tout au long de cet article tel qu'il apparaît dans les documents de planification.

5. Les planificateurs utilisent peu le terme paysage;ils lui préfèrent celui site employé principalement dans son acception géographique.

6. Schéma directeur d'aménagement et d'urbanisme de la région parisienne

7. Bernard Lassus, Pierre Dauvergne, Hugues Lambert, Bernard Fischesser, Jacques Sgard, Michel Viollet, pour ne citer qu'eux.

8. Création de la revue Landscape Ecology en 1987, inscription au CNRS en 1989.

9. Les cultures spécialisées sont repérées sur le schéma directeur comme « des pôles et des axes économiques » à renforcer.

10. Identifiées dés la fin des années 1970 pour leur richesse écologique, les basses vallées angevines sont l'objet depuis 1993 d'une dynamique forte de gestion agri-environnementale (MAE).

11. Les planificateurs cherchent à résoudre ce paradoxe en proposant d'aider financièrement les agriculteurs pour « l'entretien des structures végétales des paysages ruraux » (SRDA : 167).

12. De 1980 à 1985, A. Ricquois sera chef de la Mission du paysage. Il deviendra quelques années plus tard directeur de l'École nationale supérieure du paysage de Versailles.

13. Cette idée sera développée maintes fois: «La consistance [de l'idée de paysage dans l'Histoire] ... dépendait de l'acuité du regard posé sur le monde, de l'intérêt tant poétique que scientifique avec lequel on se tournait vers la nature " (Hervé Brunon, dans Rambault, 2009 : 174).

14. Le suivi de la genèse du SCoT et l'enquête menée auprès des acteurs qui l'ont élaboré a montré que le projet de «trame verte " promu par le Grenelle de l'Environnement au niveau national va conduire dès 2007 les acteurs locaux (élus et techniciens) à corriger, compléter et revoir leur projet de SCoT en intégrant explicitement la question de la biodiversité.

15. Seul un chargé de mission de l'AURA réalisa en interne un diagnostic des cônes de vision à partir des voies de communication sur le territoire du SCoT.

16. Selon un chargé de mission, impliqué dans le pilotage du SCoT, l'ouvrage «Au printemps des paysages » de H. et O. Décamps, 2004, a été « la » référence pour fonder et légitimer le projet de trame verte et bleue.

\section{RÉSUMÉS}

Cet article vise à suivre le cheminement de l'idée de continuité naturelle à travers le vocabulaire, ses significations et ses usages, dans les documents d'urbanisme de deux études de cas en 
contextes provincial et parisien (agglomération d'Angers et île-de-France, plus précisément la Plaine de Versailles). La question de la contribution de la pensée du paysage à l'idée de trame verte est au cœur de la réflexion proposée. On cherche en particulier à repérer le recours à la notion de paysage en mettant en exergue ses rapports avec les autres objets ou modèles fondateurs de l'action publique sur les trames vertes. Cela conduit aussi à constater la montée d'un processus de naturalisation: de plus en plus de types d'espaces, sont reconnus comme « nature » dans cette mise en réseau.

Planning greenways in urban areas : find an agreement between landscape architecture and ecology. This paper aims at following the progress of the idea of natural continuity through the vocabulary, its meanings and its manners, in the documents of urban planning of two case studies in provincial and Parisian contexts (metropolitan area of Angers and Plain of Versailles). The question of the contribution of the landscape thinking to the idea of greenway is central. We try in particular to spot the recourse to the notion of landscape by highlighting its connections with the other objects or founding models of the public action on greenways. It also leads to notice the dominion of a process of naturalization: more and more types of spaces, are recognized as "nature" in the greenways.

\section{INDEX}

Keywords : landscape thinking, landscape architecture, ecological continuity, urban planning, urbanism document

Mots-clés : pensée du paysage, paysagisme, continuités écologiques, projet urbain, planification, document d'urbanisme

\section{AUTEURS}

\section{MONIQUE TOUBLANC}

Monique Toublanc est Maître de conférences ENSP-LAREP, docteur en sociologie de l'École des Hautes Études en Sciences Sociales ; publications sur les politiques publiques paysagères (agrienvironnementale, rebocagement, restauration des paysages ruraux en déprise) ; programmes ANR Trame Verte Urbaine, et Paysage et Développement Durable du MEEDD sur la qualification des paysages périurbains par leurs habitants. Monique.toublanc@wanadoo.fr

\section{SOPHIE BONIN}

Sophie Bonin est Maître de conférences, ENSPV-LAREP, docteur en géographie de l'université Paris 1 sur les paysages du fleuve Loire; publications sur les rapports entre grandes infrastructures (barrage) et habitants ; programmes ANR Trame Verte Urbaine, et Paysage et Développement Durable du MEEDD sur la qualification des paysages périurbains par leurs habitants.sofie.bonin@free.fr 